Letter

\title{
Adhesive, Transparent Tannic Acid@ Sulfonated Lignin-PAM Ionic Conductive Hydrogel Electrode with Anti-UV, Antibacterial and Mild Antioxidant Function
}

\author{
Qinhua Wang, Hui Zhang, Xiaofeng Pan, Xiaojuan Ma*(D), Shilin Cao * and Yonghao $\mathrm{Ni}^{*}$ \\ College of Material Engineering, Fujian Agriculture and Forestry University, Fuzhou 350002, China; \\ fafuqhw@163.com (Q.W.); 17862266728@163.com (H.Z.); 1171092015@fafu.edu.cn (X.P.) \\ * Correspondence: 1212juanjuan@163.com (X.M.); scutcs1@163.com (S.C.); yonghao@unb.ca (Y.N.)
}

Received: 29 October 2019; Accepted: 9 December 2019; Published: 10 December 2019

check for updates

\begin{abstract}
Inspired by mussel adhesion chemistry and ion electronics, a novel $\mathrm{Ca}^{2+}$-tannic acid@ sulfonated lignin-polyacrylamide (TA@SL-PAM) hydrogel was prepared via $\mathrm{Ca}^{2+}$-TA@SL composites and the PAM system, where a $\mathrm{Ca}^{2+}$-TA@SL composite was fabricated via TA doping with SL and the subsequent adsorption of $\mathrm{Ca}^{2+}$. The properties of the hydrogel were thoroughly investigated and the hydrogel was presented as multifunctional. The introduction of $\mathrm{Ca}^{2+}$-TA@SL composites endowed the hydrogel with excellent conductivity, adhesion and ultraviolet (UV) resistance, and improved antioxidant and antibacterial properties. More importantly, the $\mathrm{Ca}^{2+}$-TA@SL-PAM hydrogel electrode could accurately detect physiological signals of human (e.g., electrocardiogram (ECG), electromyography (EMG).
\end{abstract}

Keywords: sulfonated lignin; tannic acid; electronic materials; functional; PAM

\section{Introduction}

Recently, conductive hydrogels (CHs) have captured the interest of scholars in the field of wearable electronics for emerging human-machine interfaces [1]. CHs integrated with excellent electrical conductivity, skin compatibility, and biocompatibility have become potential candidates for flexible medical electronics [2]; for example, flexible electrodes can detect physiological signals in humans. For the construction of visual medical products, it is urgently required that the $\mathrm{CHs}$ have great conductivity, adhesion, and transparency [3]. Moreover, the antibacterial and antioxidant properties are advantageous to $\mathrm{CHs}$ in preparing static electrodes suitable for long-term physiological monitoring. More importantly, ultraviolet (UV) protection seems to be essential because people are extremely susceptible to UV radiation from sunlight [4]. Unfortunately, it is difficult to prepare a hydrogel combing multi-functional properties simultaneously.

Here, inspired by mussel adhesion chemistry and ion electronics, we tried to use tannic acid (TA), a dopamine-like substance, to be doped with sulfonated lignin (SL) to construct composites base with adhesive properties. To further enhance the conductivity, $\mathrm{Ca}^{2+}\left(\mathrm{CaCl}_{2}\right)$ was introduced into the composite through chelation. As far as we know, TA with a large amount of catechol structures can adsorb biocompatible $\mathrm{Ca}^{2+}$.

Subsequently, the above $\mathrm{Ca}^{2+}$-TA@SL composites were incorporated into the polyacrylamide (PAM) hydrogel network, which can endow the hydrogel with great adhesion and conductivity. Meanwhile, TA and SL can impart the hydrogel with anti-UV and mild anti-bacterial ability. In addition, the TA can enhance the antioxidant properties of hydrogel. Importantly, the $\mathrm{Ca}^{2+}{ }_{-}$TA@SL-PAM CHs, as a non-invasive adhesive electrode, can achieve the accurate capture of human ECG and EMG 
signals. We believe that biomass-based $\mathrm{CHs}$ can provide more application ideas in the field of human-machine interfaces.

\section{Experimental}

Lignin was separated from the black liquor of radiated pine Kraft pulping; SL was prepared via lignin sulfonation [5]. TA@SL composite was prepared via tannic acid (TA) doping with sulfonated lignin (SL); thereafter, $\mathrm{Ca}^{2+}$ was absorbed onto the TA@SL composite. Next, acrylamide (AM), ammonium persulfate (APS), N, N'-Methylene bisacrylamide (BIS), and tetramethylethylenediamine (TMEDA) were added to the $\mathrm{Ca}^{2+}$-TA@SL suspension to form $\mathrm{Ca}^{2+}$-TA@SL-PAM hydrogel. The detailed preparation procedure and composition of the $\mathrm{Ca}^{2+}$-TA@SL-PAM hydrogels were listed in Table S1 (Supplementary Materials).

The morphology of the SL, TA@SL and $\mathrm{Ca}^{2+}$-TA@SL-PAM hydrogel were observed by field-emission scanning electron microscopy (Nova Nano SEM 230, Brno, Czech Republic), the element distribution of TA@SL was observed by Octane Elect EDS (EDAX, Mahwah, NJ, USA). The visible $(550 \mathrm{~nm})$ and UV $(365 \mathrm{~nm})$ transmittance of the PAM and $\mathrm{Ca}^{2+}$-TA@SL-PAM hydrogels with $3 \mathrm{~mm}$ thicknesses were measured using an optical transmittance meter (LS108H, Shenzhen, China). The hydrogel conductivity was measured by an LCR meter. The conductivity $(\sigma)$ is calculated by $\sigma$ $=\mathrm{d} / R S$, in which $R$ is the resistance, and $\mathrm{d}$ and $S$ are the hydrogel' geometry factors corresponding to the length and cross-sectional area, respectively. Detailed antibacterial and antioxidant assay of $\mathrm{Ca}^{2+}$-TA@SL-PAM hydrogel can be found in the Supplementary Materials. The adhesive strength (fresh pigskin) of PAM-5 hydrogel was tested via the lap-shear testing method [5].

The PAM-5 hydrogel was cut into cubes and then adhered to specific skin locations. The ECG (electrocardiograph) and EMG (electromyography) signals of volunteer were collected using a multi-channel bio-signal acquisition and process system (RM6240CD, Chengdu, China).

\section{Results and Discussion}

Figure 1a displays the complete synthetic route for the Ca ${ }^{2+}$-TA@SL-PAM hydrogel. Figure 1b shows the rice-like structure of SL. TA was doped with SL under alkaline conditions and the TA@SL composite was obtained (Figure 1c). Elemental analysis indicated that the characteristic S elements from the SL sulfonate groups were uniformly distributed on the TA@SL composite. Figure 1d demonstrates that the synthesized TA@SL particles contain more negatively charged groups than pure SL and TA particles. Therefore, the changes in the zeta potential of the various particles also confirmed the successful functionalization and assembly of the TA@SL composite. Then, $\mathrm{Ca}^{2+}$ was successfully absorbed onto the TA@SL composite through complexation with catechol groups on TA@SL composites [6]. Finally, AM, APS, MBA, and TMEDA were added to the system for in situ polymerization to form the $\mathrm{Ca}^{2+}$-TA@SL-PAM hydrogel. Figure 1e and elemental analysis (Figure 1f) present that TA@SL particles are evenly distributed on the surface of the nanocomposite hydrogel, which may provide a structural basis for the subsequent functionalization of the hydrogel. 


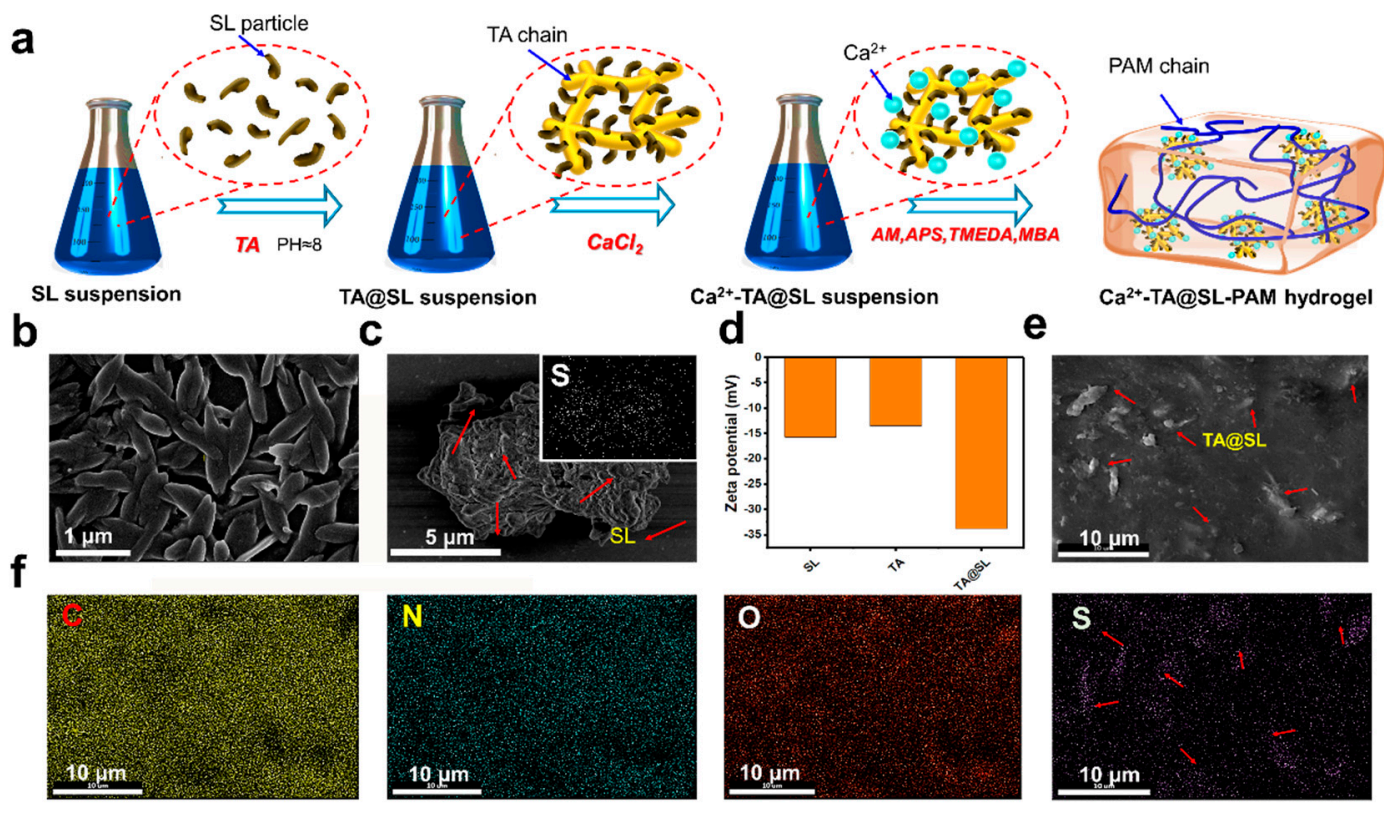

Figure 1. (a) Synthesis route of $\mathrm{Ca}^{2+}$-TA @SL-PAM hydrogel. SEM of (b) SL particles and (c) TA@SL composites and distribution of S elements on the TA@SL composite. (d) Zeta potentials of SL, TA, and TA@SL particles. (e) Distribution of TA@SL composites on the surface of $\mathrm{Ca}^{2+}$-TA@SL-PAM hydrogel.

(f) Elemental distribution mapping of the $\mathrm{Ca}^{2+}$-TA@SL-PAM hydrogel surface.

Figure 2a presents the synergy effects of the different components on the Ca ${ }^{2+}$-TA@SL-PAM hydrogel, making the resultant hydrogel have enhanced conductivity, adhesion, transparency, antioxidant, antibacterial, and anti-UV properties. As shown in Figure 2b, the raw PAM hydrogel is transparent and the transmittance was above $92 \%$. Unfortunately, it has a transmittance of $91 \%$ at $365 \mathrm{~nm}$ and is substantially incapable of filtering UV radiation. With an increase of TA content on the TA@SL composite, the UV transmission rate of TA@SL-PAM hydrogel decreased from 20\% to nearly $0 \%$. Meanwhile, the transmittance $(550 \mathrm{~nm}$ ) of the hydrogel remained above $72 \%$. For instance, the palm prints are clearly seen through the PAM-5 hydrogel. The anti-UV effect of the hydrogel is mostly derived from the absorption of UV light by the benzene ring (from SL and TA) [4]. Figure 2c shows that the TA@SL-PAM hydrogel can use conductive $\mathrm{Ca}^{2+}$ to achieve the circuit connectivity $(0.91 \mathrm{~S} / \mathrm{m})$. The interaction of catechol groups on the TA and $\mathrm{Ca}^{2+}$ inspired by mussel adhesion chemistry can impart adhesion to the hydrogel [7]. As presented in Figure 2d, PAM-5 hydrogels can be bonded to different materials such as skin, foam, plastic, iron, rubber, and glass. As revealed in Figure 2e, the PAM hydrogel shows no effect on scavenging staphylococcus aureus (S. aureus), while the SL based PAM hydrogel and $\mathrm{Ca}^{2+}$-TA@SL-PAM hydrogel can scavenge some of the S. aureus. Notably, PAM-5 hydrogel can even achieve a $98 \%$ S. aureus killing rate. Figure $2 \mathrm{f}$ indicates the UV-Vis absorption curve of the 1,1-diphenyl-2-picrylhydrazyl (DPPH) free radical solution in the control group and $\mathrm{Ca}^{2+}$-TA@SL-PAM hydrogel with different TA@SL content. Due to the presence of odd electrons, the DPPH radical generates a strong absorption peak at $517 \mathrm{~nm}$ [8]. However, the coordination of electrons with the hydrogen atoms in the antioxidant results in a decrease in the intensity of the absorption peak. It is clearly seen that the absorption intensity decreases with increasing TA@SL content in $\mathrm{Ca}^{2+}$-TA@SL-PAM hydrogel. Compared with the control group, the DPPH scavenging rate even reached $75 \%$ when the TA@SL content reached $16 \mathrm{mg} / \mathrm{mL}$ (Figure $2 \mathrm{~g}$ ). These results demonstrate that the $\mathrm{Ca}^{2+}$-TA@SL-PAM hydrogel has effective antioxidant properties. Generally, the antioxidant ability of the hydrogel is derived from the action of TA and lignin; the phenolic hydroxyl group on TA and SL can reduce the free DPPH radical to diphenyl-picrylhydrazine [9]. 
a

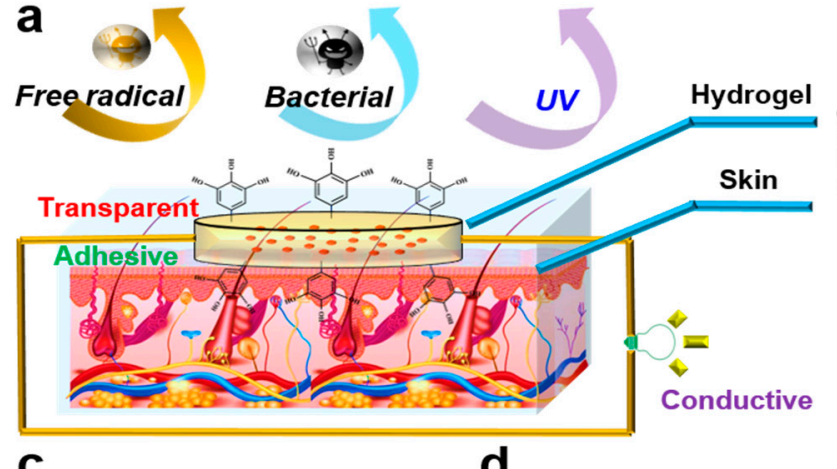

C

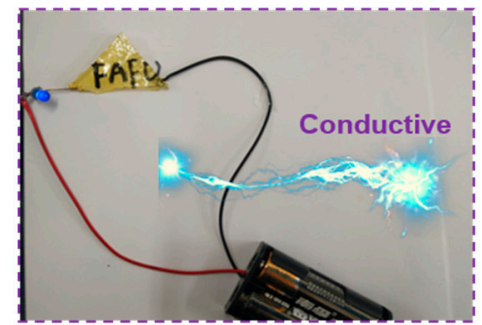

e

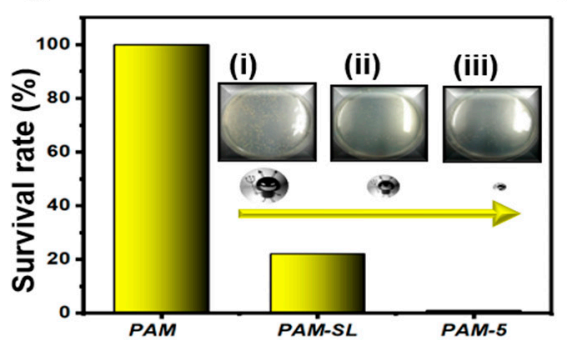

b
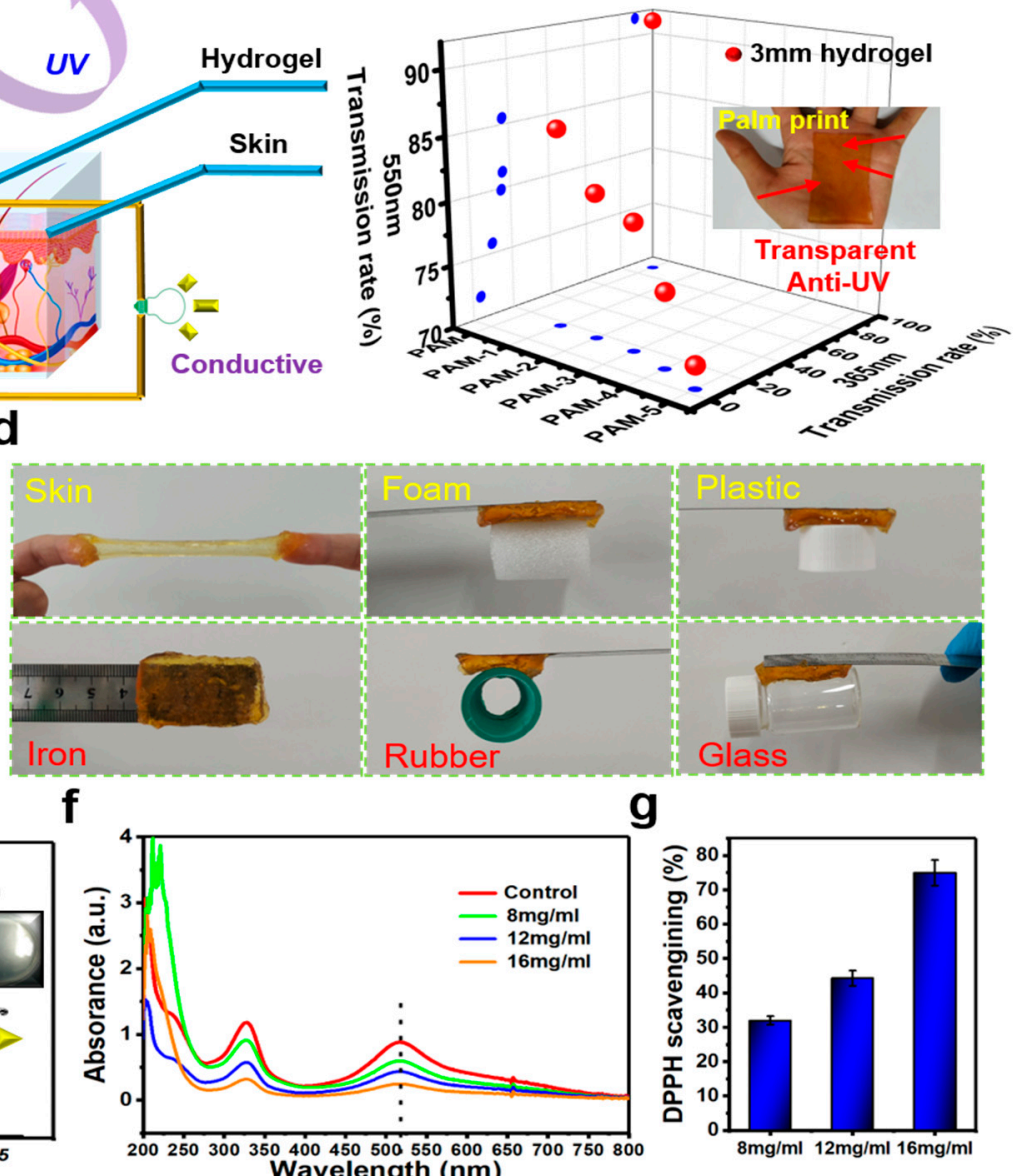

Figure 2. (a) Cartoon illustration of the multifunctional $\mathrm{Ca}^{2+}-\mathrm{TA} @ S L-P A M$ hydrogel. (b) The transmittance of $\mathrm{Ca}^{2+}$-TA@SL-PAM hydrogel (c) PAM-5 can be used as a circuit wire. (d) PAM-5 can be attached to different materials. (e) Survival rate of $S$. aureus grown on different hydrogels. (f) Absorbance change of DPPH when exposed to different hydrogels. (g) DPPH scavenging rate.

Biocompatible $\mathrm{Ca}^{2+}$ have a greater advantage in the preparation of non-invasive electronics [10]. Herein, the suitability of ion-conductive $\mathrm{Ca}^{2+}$-TA@SL-PAM hydrogel electrodes was evaluated in detail. As presented in Figure 3a, the hydrogel could be firmly adhered on the skin and the even arm shook vigorously (the adhesion strength between hydrogel and fresh pig skin is $4.31 \mathrm{KPa}$ ). Moreover, the adhesive hydrogel could be completely peeled off and had no residue on the arm. Importantly, the adhesive electrodes attached to the palm muscle of the female volunteer's arm achieved accurate collection of epidermal electrical signal changes caused by the volunteers' arm-clamping, relaxation, and straightening muscle switching (Figure 3b). Similarly, volunteers' complete ECG and heart rate could also be accurately monitored by the adhesive electrodes (Figure 3c) [5]. Adhesive hydrogel can increase the service life of the electrode and prevent it from falling off the skin, thereby affecting the stability of the signal. 


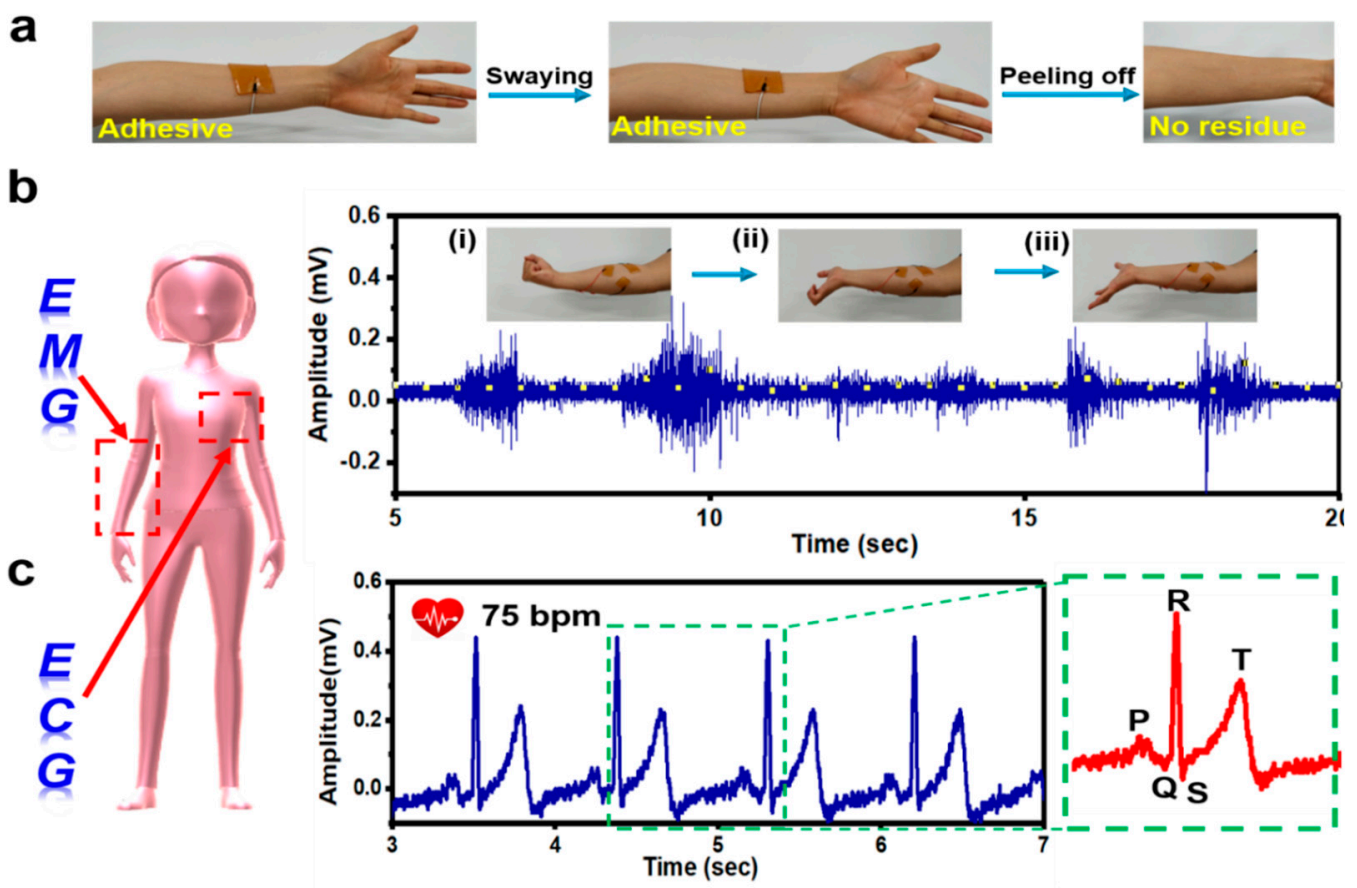

Figure 3. (a) PAM-5 adheres tightly to the author's skin and shakes sharply without falling off. (b) The electrode collects variations in EMG signals. (c) The electrode can monitor ECG signals of human.

\section{Conclusions}

A novel PAM based ionic conductive hydrogel electrode was successfully developed-inspired by mussel and ion electronics. The TA@SL composite application endowed the hydrogel with excellent adhesion, UV resistance, antioxidant, and moderate antibacterial properties, but without the destruction of transparency. The $\mathrm{Ca}^{2+}$ introduction imparted the hydrogel with electrical conductivity. The fabricated hydrogel electrode could accurately collect the physiological signals from the human, including EMG and ECG. Considering the prominent properties and quick feedback of the human motion, the $\mathrm{Ca}^{2+}$-TA@SL-PAM hydrogel electrode has great potential in visual medical electronics.

Supplementary Materials: The following are available online at http://www.mdpi.com/1996-1944/12/24/4135/s1, Table S1: Detailed composition of various hydrogels.

Author Contributions: Conceptualization, Q.W. and X.P.; methodology, H.Z.; formal analysis, X.P.; investigation, Q.W. and H.Z.; writing-original draft preparation, Q.W.; writing-review and editing, X.M. and S.C.; supervision, X.M., S.C.; project administration, Y.N.; funding acquisition, Y.N.

Funding: This research was funded by the National Natural Science Foundation of China (31770632), funding from Fujian Education Department for the "Excellent Talents Support Plan in Fujian Universities" and Innovation Fund from Fujian Agriculture and Forestry University (CXZX2017296 and CXZX2017037).

Conflicts of Interest: The authors declare no conflict of interest. The funders had no role in the design of the study; in the collection, analyses, or interpretation of data; in the writing of the manuscript, or in the decision to publish the results.

\section{References}

1. Yuk, H.; Lu, B.; Zhao, X. Hydrogel bioelectronics. Chem. Soc. Rev. 2019, 48, 1642-1667. [CrossRef] [PubMed]

2. Sun, J.-Y.; Keplinger, C.; Whitesides, G.M.; Suo, Z. Ionic skin. Adv. Mater. 2014, 26, 7608-7614. [CrossRef] [PubMed]

3. Han, L.; Yan, L.; Wang, M.; Wang, K.; Fang, L.; Zhou, J.; Fang, J.; Ren, F.; Lu, X. Transparent, Adhesive, and Conductive Hydrogel for Soft Bioelectronics Based on Light-Transmitting Polydopamine-Doped Polypyrrole Nanofibrils. Chem. Mater. 2018, 30, 5561-5572. [CrossRef] 
4. Pan, X.; Wang, Q.; Guo, R.; Ni, Y.; Liu, K.; Ouyang, X.; Chen, L.; Huang, L.; Cao, S.; Xie, M. An integrated transparent, UV-filtering organohydrogel sensor via molecular-level ion conductive channels. J. Mater. Chem. A 2019, 7, 4525-4535. [CrossRef]

5. Wang, Q.; Pan, X.; Lin, C.; Lin, D.; Ni, Y.; Chen, L.; Huang, L.; Cao, S.; Ma, X. Biocompatible, self-wrinkled, antifreezing and stretchable hydrogel-based wearable sensor with PEDOT: sulfonated lignin as conductive materials. Chem. Eng. J. 2019, 370, 1039-1047. [CrossRef]

6. Rahim, M.A.; Björnmalm, M.; Bertleff-Zieschang, N.; Ju, Y.; Mettu, S.; Leeming, M.G.; Caruso, F. Multiligand Metal-Phenolic Assembly from Green Tea Infusions. ACS Appl. Mater. Inter. 2018, 10, 7632-7639. [CrossRef] [PubMed]

7. Shao, C.; Wang, M.; Meng, L.; Chang, H.; Wang, B.; Xu, F.; Yang, J.; Wan, P. Mussel-Inspired Cellulose Nanocomposite Tough Hydrogels with Synergistic Self-Healing, Adhesive, and Strain-Sensitive Properties. Chem. Mater. 2018, 30, 3110-3121. [CrossRef]

8. Ge, W.; Cao, S.; Shen, F.; Wang, Y.; Ren, J.; Wang, X. Rapid self-healing, stretchable, moldable, antioxidant and antibacterial tannic acid-cellulose nanofibril composite hydrogels. Carbohyd. Polym. 2019, 224, 115147. [CrossRef] [PubMed]

9. Li, X.; Liu, Y.; Yu, Y.; Chen, W.; Liu, Y.; Yu, H. Nanoformulations of quercetin and cellulose nanofibers as healthcare supplements with sustained antioxidant activity. Carbohyd. Polym. 2019, 207, 160-168. [CrossRef] [PubMed]

10. Tao, X.; Liao, S.; Wang, S.; Wu, D.; Wang, Y. Body Compatible Thermometer Based on Green Electrolytes. ACS Sensors 2018, 3, 1338-1346. [CrossRef] [PubMed]

(C) 2019 by the authors. Licensee MDPI, Basel, Switzerland. This article is an open access article distributed under the terms and conditions of the Creative Commons Attribution (CC BY) license (http://creativecommons.org/licenses/by/4.0/). 